\title{
On the origin of stars with and without planets
}

\section{$T_{c}$ trends and clues to Galactic evolution $\star, \star \star$}

\author{
V. Zh. Adibekyan ${ }^{1}$, J. I. González Hernández ${ }^{2,3}$, E. Delgado Mena ${ }^{1}$, S. G. Sousa ${ }^{1,2,4}$, N. C. Santos ${ }^{1,4}$, G. Israelian ${ }^{2,3}$, \\ P. Figueira ${ }^{1}$, and S. Bertran de Lis ${ }^{2,3}$ \\ ${ }^{1}$ Centro de Astrofísica da Universidade do Porto, Rua das Estrelas, 4150-762 Porto, Portugal \\ e-mail: Vardan. Adibekyan@astro.up.pt \\ 2 Instituto de Astrofísica de Canarias, 38200 La Laguna, Tenerife, Spain \\ 3 Departamento de Astrofísica, Universidad de La Laguna, 38206 La Laguna, Tenerife, Spain \\ ${ }^{4}$ Departamento de Física e Astronomia, Faculdade de Ciências da Universidade do Porto, 4169-007 Porto, Portugal
}

Received 15 January 2014 / Accepted 25 March 2014

\section{ABSTRACT}

\begin{abstract}
We explore a sample of 148 solar-like stars to search for a possible correlation between the slopes of the abundance trends versus condensation temperature (known as the $T_{\mathrm{c}}$ slope) with stellar parameters and Galactic orbital parameters in order to understand the nature of the peculiar chemical signatures of these stars and the possible connection with planet formation. We find that the $T_{\mathrm{c}}$ slope significantly correlates (at more than $4 \sigma$ ) with the stellar age and the stellar surface gravity. We also find tentative evidence that the $T_{\mathrm{c}}$ slope correlates with the mean galactocentric distance of the stars $\left(R_{\text {mean }}\right)$, suggesting that those stars that originated in the inner Galaxy have fewer refractory elements relative to the volatiles. While the average $T_{\mathrm{c}}$ slope for planet-hosting solar analogs is steeper than that of their counterparts without planets, this difference probably reflects the difference in their age and $R_{\text {mean }}$. We conclude that the age and probably the Galactic birth place are determinant to establish the star's chemical properties. Old stars (and stars with inner disk origin) have a lower refractory-to-volatile ratio.
\end{abstract}

Key words. stars: abundances - stars: atmospheres - planetary systems - stars: kinematics and dynamics

\section{Introduction}

Despite the huge progress in developing instrumentation and observational techniques during the past decade, the study of extrasolar planets' properties via direct observations is still a very difficult task, and the precise study and characterization of known extroplanets cannot be dissociated from the study of planet host stars.

The connection between stellar and planetary properties has been widely explored. In particular, the very first correlation observed in this field of research, that of giant-planet - metallicity (e.g., Gonzalez 1997; Santos et al. 2001, 2004; Fischer \& Valenti 2005; Sousa et al. 2011), was one of the most important constraints on planet formation theories (e.g., Mordasini et al. 2009). Afterwards it was shown that not only does the presence of planets correlate with metallicity (usually abundance of iron), but planet-host stars also show a systematic enhancement of other elements (e.g., $\alpha$-elements - Haywood 2008a; Adibekyan et al. 2012a,b). More recently, several studies have revealed that stellar metallicity also plays an important role in the architecture of

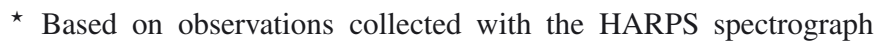
at the 3.6-m telescope (072.C-0488(E)), installed at the La Silla Observatory, ESO (Chile), with the UVES spectrograph at the 8-m Very Large Telescope program IDs: 67.C-0206(A), 074.C-0134(A), 075.D0453(A), installed at the Cerro Paranal Observatory, ESO (Chile), and with the UES spectrograph at the 4.2-m William Herschel Telescope, installed at the Spanish Observatorio del Roque de los Muchachos of the Instituto de Astrofísica de Canarias, on the island of La Palma.

$\star \star$ Appendix A is available in electronic form at

http://www. aanda.org planetary orbits (e.g., Dawson \& Murray-Clay 2013; Beaugé \& Nesvorný 2013; Adibekyan et al. 2013b).

Naturally, this connection is bidirectional: not only do stellar properties play an important role in planet formation and evolution, but the planet formation can also have an impact on stellar properties. As an example, Israelian et al. (2009) and Delgado Mena et al. (2014) find strong evidence that solar analogs with planets show enhanced Li depletion compared to their non-host counterparts. The studies mentioned above are not the only ones. Recently, Meléndez et al. (2009) have claimed that the Sun shows a deficiency in refractory elements with respect to other solar twins probably because they were trapped in the terrestrial planets in our solar system. The same conclusion has also been reached by Ramírez et al. (2009) who analyzed 64 solar twins and analogs. However, recent results by (González Hernández et al. 2010, 2013 - GH10,13) strongly challenge the relation between the presence of planets and the abundance peculiarities of the stars. Other works also have examined this possible connection between the chemical peculiarities and formation of planets (e.g., Smith et al. 2001; Ecuvillon et al. 2006; Gonzalez et al. 2010; Gonzalez 2011; González Hernández et al. 2011; Schuler et al. 2011; Ramírez et al. 2014).

In this Letter, we explore the origin of the possible trend observed between $[X / \mathrm{H}]($ or $[X / \mathrm{Fe}])$ and condensation temperature $\left(T_{\mathrm{c}}\right)$ using a sample of 148 solar-like stars.

\section{Data}

Our initial sample is a combination of two samples of solar analogs (95 stars) and "hot" analogs (61 stars) taken from GH10,13. We have cross-matched this sample with the 
Table 1. Significance levels of the obtained correlations.

\begin{tabular}{lcccc}
\hline \hline Parameter & Cor. coeff & $\begin{array}{c}\text { Significance } \\
\text { All }\end{array}$ & $\begin{array}{c}\text { Cor. coeff } \\
\text { Solar analogs }\end{array}$ & $\begin{array}{c}\text { Significance } \\
\text { Age }\end{array}$ \\
\hline $\log g$ & 0.35 & $\mathbf{4 . 3 7}$ & 0.60 & $\mathbf{4 . 5 7}$ \\
{$[\mathrm{Fe} / \mathrm{H}]$} & -0.50 & $\mathbf{6 . 0 1}$ & -0.73 & $\mathbf{5 . 5 7}$ \\
$T_{\text {eff }}$ & -0.14 & 1.73 & 0.00 & 0.05 \\
\hline
\end{tabular}

Geneva-Copenhagen Survey sample (GCS- Nordström et al. 2004), for which Casagrande et al. (2011) provides the Galactic orbital parameters, the space velocity components, and the ages of 148 of the stars considered in our study ${ }^{1}$. Fifty-seven of these stars are planet hosts, while for the remaining 91 no planetary companion has been detected up to now.

The stellar atmospheric parameters and the slopes of the $\Delta[X / \mathrm{Fe}]_{\text {SUN-star }}$ versus $T_{\mathrm{c}}$ were derived using very high-quality HARPS spectra ${ }^{2}$. Twenty-five elements from $C(Z=6)$ to Eu $(Z=63)$ have been used for this analysis. These slopes are corrected for the Galactic chemical evolution trends as discussed in GH10,13.

The stars in the sample have effective temperatures $5604 \mathrm{~K} \leq$ $T_{\text {eff }} \leq 6374 \mathrm{~K}$, metallicites $-0.29 \leq[\mathrm{Fe} / \mathrm{H}] \leq 0.38 \mathrm{dex}$, and surface gravities $4.14 \leq \log g \leq 4.63$ dex. Throughout the Letter we defined solar analogs as stars with; $T_{\text {eff }}=5777 \pm 200 \mathrm{~K}$; $\log g=4.44 \pm 0.20 \mathrm{dex} ;[\mathrm{Fe} / \mathrm{H}]=0.0 \pm 0.2$ dex. Solar twins are defined as with; $T_{\text {eff }}=5777 \pm 100 \mathrm{~K} ; \log g=4.44 \pm 0.10 \mathrm{dex}$; $[\mathrm{Fe} / \mathrm{H}]=0.0 \pm 0.1$ dex. Fifteen out of 58 solar analogs in this sample are known to be orbited by planets and three out of 15 solar twins are planet hosts.

We would like to point out that stellar parameters derived in GH10,13 and in Casagrande et al. (2011) are in perfect agreement $\left(\Delta T_{\text {eff }}=-8 \pm 50 \mathrm{~K}, \Delta \log g=0.06 \pm 0.07 \mathrm{dex}\right.$, and $\Delta[\mathrm{Fe} / \mathrm{H}]=-0.02 \pm 0.06 \mathrm{dex})$, which means that the ages derived in Casagrande et al. (2011) are indeed coherent with the rest of the observables.

\section{Correlations with $T_{\mathrm{c}}$ slope}

We searched for possible correlations between the $T_{\mathrm{c}}$ slope and, in turn, atmospheric parameters and also Galactic orbital parameters and age, in order to understand which is/are the main factor(s) responsible for the abundance trends with $T_{\mathrm{c}}$.

\section{1. $T_{\mathrm{C}}$ slope against stellar parameters and age}

In Fig. A. 1 we show the relation between the $T_{\mathrm{c}}$ slopes and $\log g$ both for the full sample and for the solar analogs exclusively. One can note that the $T_{\mathrm{c}}$ slopes strongly correlate with the $\log g$. To evaluate the significance of the correlation we performed a simple bootstrapped Monte Carlo test. For more details about the test we refer the reader to Figueira et al. (2013) and Adibekyan et al. (2013a). The correlation coefficients and the significance levels (z-scores) of the correlations are presented in Table 1. The same table shows that $T_{\mathrm{c}}$ slopes do not significantly correlate with other stellar parameters. The $T_{\mathrm{c}}$ slope versus $[\mathrm{Fe} / \mathrm{H}]$ and $T_{\text {eff }}$ plots are shown in Fig. A.2.

The observed significant relation between the surface gravity and $T_{\mathrm{c}}$ slope means that the chemical abundance trends depend

\footnotetext{
1 Throughout the paper, BASTI expectation ages are used as suggested by Casagrande et al. (2011).

2 Zero slope means solar chemical composition, and a positive slope corresponds to a smaller refractory-to-volatile ratio compared to the Sun.
}

either on the evolutionary stage of the star or on its age ${ }^{3}$. In Fig. 1 we plot the $T_{\mathrm{c}}$ slope against the stellar age. The plot and Table 1 clearly show that the correlation with age is quite significant and confirms the result obtained for the surface gravity: old stars are more depleted in refractory elements (lower refractoryto-volatile ratios) than their younger counterparts.

For FGK dwarf stars in the main sequence one does not expect significant changes in their atmospheric chemical abundances with age. This means that the observed correlation between the $T_{\mathrm{c}}$ slope and age probably reflects the chemical evolution in the Galaxy. We note that this is the simplest assumption we can make based on our limited current knowledge of stellar evolution, and we caution the reader that might be other effects that could severely affect the composition of stars as a function of age. For example, in the solar wind, elements with low first ionization potentials (FIP) are about four times more abundant than in the photosphere of the sun (e.g., Geiss 1998; Raymond 1999). However, this so-called FIP-effect, which will depend on age and has significant effect on coronal abundances (Wood \& Linsky 2006), is not expected to affect the photospheric abundances.

In this context it is worth mentioning the work of Meléndez et al. (2009), where the authors discussed several possible explanations of the solar "peculiar" abundances when compared to the solar twins. Most of the discussed possible effects (e.g., supernova pollution, early dust separation, etc.) however are not expected to have a dependence on age and can indeed be responsible for peculiarities in chemical composition for individual cases (stars). In fact the median age of their comparison solar-twin stars was of $4.1 \mathrm{Gyr}$, a very similar to that of the Sun. The authors also discussed the Galactic evolution effects, taking into account the possibility that the Sun may have migrated from an inner Galactic orbit (Wielen et al. 1996), but ended up not considering it as a probable explanation.

\section{2. $T_{\mathrm{c}}$ slope and Galactic orbital parameters}

Several studies have shown that the mean of the apo- and pericentric distances $\left(R_{\text {mean }}\right)$ are good indicators of the stellar birthplace (e.g., Grenon 1987; Edvardsson et al. 1993; Nordström et al. 1999; Rocha-Pinto et al. 2004; Bensby et al. 2014). Haywood (2008b) shows that orbital parameters $\left(R_{\text {mean }}\right)$ of the metal-poor and metal-rich thin disk stars are significantly different from those of the main thin-disk population, and an outer and inner galactic disk origin was suggested for them both, respectively. A word of caution should be added here, if the radial migration proposed by Sellwood \& Binney (2002) ${ }^{4}$ is efficient, then using $R_{\text {mean }}$ as a proxy for the birthplace of a star could be dubious. However, Haywood et al. (2013) cast doubt upon the efficiency of churning for contaminating the solar neighborhood.

In Fig. 2 we study the dependence of the $T_{\mathrm{c}}$ slopes on $R_{\text {mean }}$, which we use as a proxy of the birth radii. Even if the correlation between the $T_{\mathrm{c}}$ slopes and $R_{\text {mean }}$ is not strong and is not significant (at $1 \sigma$ level), one can see that most of the stars with the lowest mean galactocentric distances have steeper average $T_{\text {c }}$ slopes $(\approx 0.071 \pm 0.065)$ than the average of the stars with $R_{\text {mean }}=8 \pm 1 \mathrm{kpc}\left(T_{\mathrm{c}} \approx-0.086 \pm 0.035\right)$. The significance of this difference is $2.1 \sigma$ if one applies two sample $t$-test. For the solar analog sample, the significance of this difference is less significant (at a level of $1.5 \sigma$ ).

\footnotetext{
3 Since a solar type star evolves from ZAMS, its log $g$ slowly increases with age along the main sequence.

4 The term "churning" was introduced in Schönrich \& Binney (2009) for this type of migration.
} 


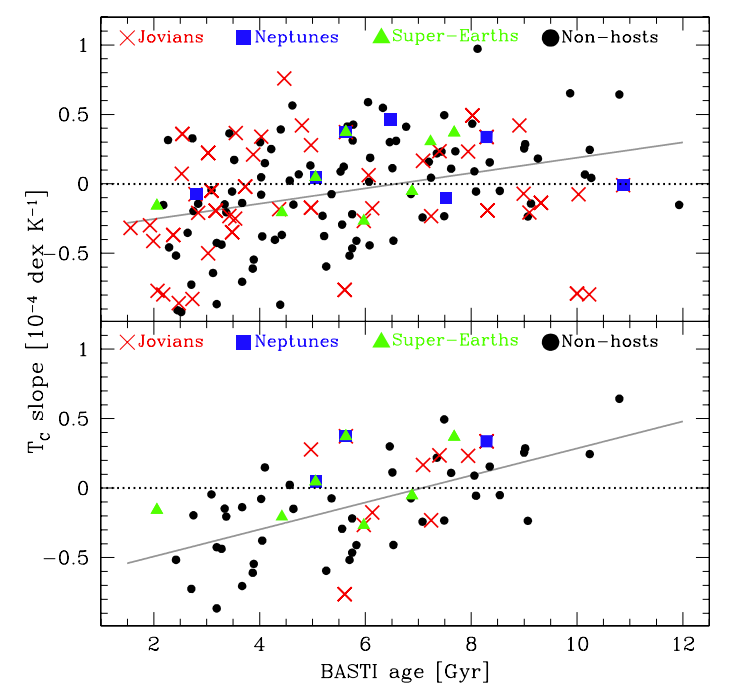

Fig. 1. $T_{\mathrm{c}}$ slopes versus ages for the full sample (top) and for the solar analogs (bottom). Gray solid lines provide linear fits to the data points.

In Fig. A.3 we show the relation between the $T_{\mathrm{c}}$ slopes and both the stellar age and the $R_{\text {mean }}$ for solar twins in our sample. As one can see, all the correlations obtained for the full sample and sample of solar analogs are also valid for the solar twins. Solar twins follow the general trends discussed in this section and in Sect. 3.1.

Rocha-Pinto et al. (2004), among other authors, have already shown that $R_{\text {mean }}$ correlates with age ${ }^{5}$ in such a way that young objects (mostly younger than one Gyr) all have $R_{\text {mean }} \approx R_{\odot}$, while older stars present a higher proportion of objects coming from different galactocentric radii. In Fig. A. 4 we plot $R_{\text {mean }}$ versus stellar age. One can note that there is a weak correlation between the two parameters (with a correlation coefficient of $\approx 0.16$ at a significance level of $\approx 2 \sigma$ ), even if not as an apparent way as in Fig. 7 of Rocha-Pinto et al. (2004), probably because we do not have very young stars in our sample. However, in Fig. A.4 one can note that old stars (older than 6-7 Gyr) from the inner Galaxy $\left(R_{\text {mean }}<7\right)$ have predominantly positive $T_{\mathrm{c}}$ slopes, while those in the solar circle have negative slopes. In addition, the same figure shows that young stars with inner $R_{\text {mean }}$ values show an overabundance of negative $T_{\mathrm{c}}$ slopes, while their older counterparts mostly have positive slopes.

To test our findings observationally, i.e., if the $T_{\mathrm{c}}$ slopes are different at different galactocentric distances $(R)$, we used the Galactic abundance gradients derived in Lemasle et al. (2008, 2013). Using the linear fits (provided by these authors) of the gradient for each elements, we derived $[X / \mathrm{Fe}]$ abundance ratios at four galactocentric radii $(6,8$, and $10 \mathrm{kpc})$. Then we plotted $[X / \mathrm{Fe}]$ against $T_{\mathrm{c}}$ (see Fig. A.5), and after performing a linear fit, we calculated the $T_{\mathrm{c}}$ slopes. From the figure it is evident that at smaller galactocentric distances, the $T_{\mathrm{c}}$ slope is steeper ${ }^{6}$ which qualitatively (not quantitatively) agrees well with what we obtained in the $T_{\mathrm{c}}$ versus $R_{\text {mean }}$ plot. We note that in Lemasle et al. (2008, 2013), the authors used young Galactic Cepheids to derive the gradients. This means that the gradients should be considered as current ones, which in turn means that they are not affected by any type of migration. Although not quantitative, this comparison shows that at a fixed age (the present day) the

\footnotetext{
5 Wielen et al. (1996) have shown that this relation can only be considered in a statistical sense.

6 We note that here one should changed the sign of the $T_{\mathrm{c}}$ slopes to be consistent with the $T_{\mathrm{c}}$ slopes derived in GH10,13.
}

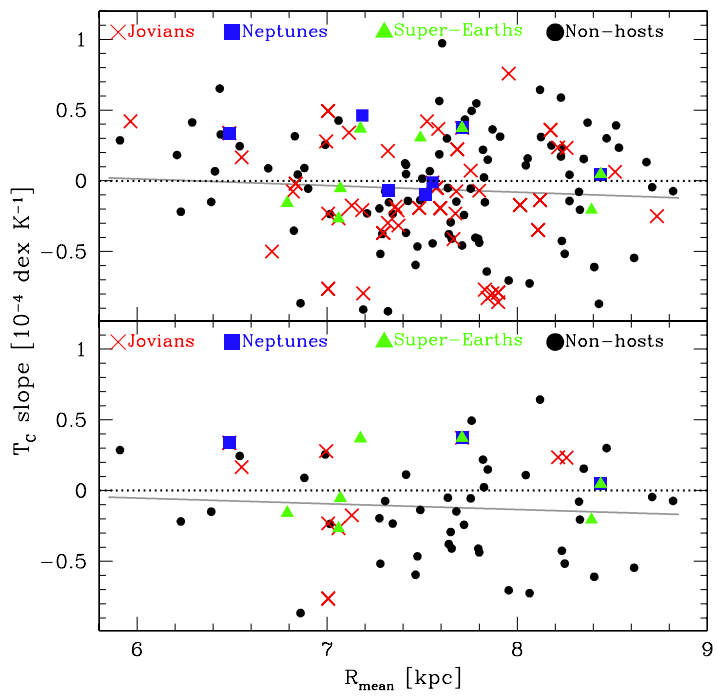

Fig. 2. $T_{\mathrm{c}}$ slopes versus $R_{\text {mean }}$ for the full sample (top) and for the solar analogs (bottom). Gray solid lines provide linear fits to the data points.

$T_{\mathrm{c}}$ correlates with the galactocentric radius. This is one more hint that $R_{\text {mean }}$ could be a relevant parameter for "single-age" populations (as already noted for the other end of the age domain explored in the paper, i.e. old stars, discussed in Fig. A.4).

The tentative correlation between $T_{\mathrm{c}}$ and the mean galactocentric distance of the stars, and the observational results of Lemasle et al. (2008), suggests that the chemical composition of the "birth place" is a factor partially responsible for the observed $T_{\mathrm{c}}$ trends. The observed (weak) correlations suggest that at a fixed time the steeper $T_{\mathrm{c}}$ slopes (lower refractory-to-volatile ratios) are associated to stars that probably have originated in the inner Galaxy. This result is in line with the recent chemical evolution models of the Milky Way invoking stellar migration (e.g., Minchev et al. 2013). Older stars are exposed to perturbations and scattering for a longer period of time and have also had more time to migrate, therefore some fraction of them originated in the inner Galaxy, while almost all the young stars have birth radii within the solar radius (Wang \& Zhao 2013; Minchev et al. 2013).

\section{3. $T_{\mathrm{C}}$ slope and planets}

In this Letter we do not develop a long discussion about the signatures of planet formation on the abundance trends since it has already been discussed in detail by GH10,13. Using the same sample they have already shown that there is no statistically significant difference in $T_{\mathrm{c}}$ slopes for planet-hosting stars (in particular for rocky planet hosts) and stars without any detected planetary companion. In fact the average $T_{\mathrm{c}}$ slope of the planet hosting stars $(-0.08 \pm 0.38)$ is even slightly smaller (the difference is not significant) than that of the non-host stars $(-0.05 \pm 0.39)$ from the full sample.

Following our definition of solar analogs, we found that the average of the $T_{\mathrm{c}}$ slope for planet hosting solar analogs is greater $(0.012 \pm 0.31)$ than that of their non-host counterparts $(-0.16 \pm 0.34)$. The Kolmogorov-Smirnov (K-S) statistics predict the $\approx 0.21$ probability $\left(P_{\mathrm{KS}}\right)$ that these two subsamples came from the same underlying distribution for $T_{\mathrm{c}}$ slope. At the same time, the same statistics predict a $P_{\mathrm{KS}} \approx 0.20$ probability that they stem from the same underlying age distributions. The latter can be seen in Fig. 1: most of the planet-hosting stars tend to be relatively old ( $\gtrsim 5$ Gyr). Moreover, planet host and non-host 
samples show a different distribution of $R_{\text {mean }}-P_{\mathrm{KS}} \approx 0.007$. As can also be seen in Fig. 2, 10 planet hosts out of $15(66 \%)$ have $R_{\text {mean }}$ smaller than $7.5 \mathrm{kpc}$ (where slopes are usually high) and only 16 out of 43 stars without detected planets (37\%) have similarly low $R_{\text {mean }}$ values. Clearly the two subsamples are not consistent with respect to the mean galactocentric distance and age. Interestingly, Haywood (2009) has already shown that (giant) planet host stars tend to have smaller $R_{\text {mean }}$ and probably originate in the inner disk, which follow the same direction as our findings.

We note that the mean value of the $T_{\mathrm{c}}$ slopes of the superEarth hosts $\left(M_{\text {planet }}<0.05 M_{\mathrm{J}}\right)$ is very close to the average value of the Jovian hosts $\left(M_{\text {planet }}>0.1 M_{\mathrm{J}}\right)$, while the average $T_{\mathrm{c}}$ slope of the three Neptunian $\operatorname{hosts}^{7}\left(0.05 M_{\mathrm{J}} \geq M_{\text {planet }} \geq 0.1 M_{\mathrm{J}}\right)$ is greater. For a more detailed comparison of the $T_{\mathrm{c}}$ slopes for planet hosts at different planetary mass regimes, we refer the reader to $\mathrm{GH} 10,13$.

These results suggest that the difference in $T_{\mathrm{c}}$ slopes observed for solar analogs with and without planets is probably due to the differences in their "birth places" and times.

\section{Summary and conclusion}

We used a sample of 148 solar-type stars from GH10,13 to explore the main factors responsible for the abundance trends with condensation temperature. For these stars the stellar atmospheric parameters and the $T_{\mathrm{c}}$ slopes were taken from the abovementioned studies, while the stellar ages, Galactic orbital parameters, and velocity components are from Casagrande et al. (2011).

Our study reveals a strong correlation between stellar ages and $T_{\mathrm{c}}$ slope: old stars show steeper slope i.e., less refractory elements relative to volatiles. The same result reflects the statistically significant correlation between $T_{\mathrm{c}}$ slopes and surface gravity: more evolved (old) stars have a lower refractory-to-volatile ratio.

Moving one step further, we found tentative evidence that the $T_{\mathrm{c}}$ slopes also correlate with the mean galactocentric distance of the stars; this suggest that stars which probably have origin in the inner Galaxy (small $R_{\text {mean }}$ ) have steeper slopes. The result fits well in the recent evolution picture of the Milky Way, showing that some fraction of old stars in the solar neighborhood might have its origin in the inner disk (e.g., Minchev et al. 2013).

Briefly exploring the possible reasons why one can see a difference in $T_{\mathrm{c}}$ slopes for planet-hosting solar analogs and solar analogs without detected planets, we found that in the current sample these two subsamples have different distributions of age and $R_{\text {mean }}$ that correlate with the $T_{\mathrm{c}}$ slope. These differences might explain the differences in $T_{\mathrm{c}}$ slope distribution, suggesting that there are no signatures of planet formation in the observed abundance trends with the condensation temperature.

We may conclude that the $T_{\mathrm{c}}$ slope depends on the age of a star at a fixed galactocentric radius. At the same time, stars with smaller galactocentric radii show stepper $T_{\mathrm{c}}$ slopes at a fixed time (age). In other words, the age and galactic birth place may determine the chemical pattern of the stars.

Acknowledgements. We gratefully thank Misha Haywood and Ivan Minchev for fruitful comments and discussion. We gratefully acknowledge the (second) anonymous referee for a very exhaustive and honest review of the manuscript that led to many constructive comments and suggestions. This work was supported by the European Research Council/European Community under the FP7 through Starting Grant agreement number 239953. V.Zh.A., S.G.S., and E.D.M are supported by grants SFRH/BPD/70574/2010, SFRH/BPD/47611/2008, and SFRH/BPD/76606/2011 from the FCT (Portugal), respectively. NCS also acknowledges support in the form of a Investigador FCT contract funded by FCT/MCTES (Portugal) and POPH/FSE (EC). G.I., S.B.L, and J.I.G.H. acknowledge financial support from the Spanish Ministry project MINECO AYA2011-29060, and J.I.G.H. also received support from the Spanish Ministry of Economy and Competitiveness (MINECO) under the 2011 Severo Ochoa Program MINECO SEV-2011-0187. P.F. is supported by the FCT and POPH/FSE (EC) through an Investigador FCT contract with application reference IF/01037/2013 and and POPH/FSE (EC) by FEDER funding through the program "Programa Operacional de Factores de Competitividade" - COMPETE.

\section{References}

Adibekyan, V. Z., Delgado Mena, E., Sousa, S. G., et al. 2012a, A\&A, 547, A36 Adibekyan, V. Z., Santos, N. C., Sousa, S. G., et al. 2012b, A\&A, 543, A89 Adibekyan, V. Z., Figueira, P., Santos, N. C., et al. 2013a, A\&A, 554, A44 Adibekyan, V. Z., Figueira, P., Santos, N. C., et al. 2013b, A\&A, 560, A51 Beaugé, C., \& Nesvorný, D. 2013, ApJ, 763, 12

Bensby, T., Feltzing, S., \& Oey, M. S. 2014, A\&A, 562, A71

Casagrande, L., Schönrich, R., Asplund, M., et al. 2011, A\&A, 530, A138

Dawson, R. I., \& Murray-Clay, R. A. 2013, ApJ, 767, L24

Delgado Mena, E., Israelian, G., González Hernández, J. I., et al. 2014, A\&A, 562, A92

Ecuvillon, A., Israelian, G., Santos, N. C., Mayor, M., \& Gilli, G. 2006, A\&A, 449,809

Edvardsson, B., Andersen, J., Gustafsson, B., et al. 1993, A\&A, 275, 101

Figueira, P., Santos, N. C., Pepe, F., Lovis, C., \& Nardetto, N. 2013, A\&A, 557, A93

Fischer, D. A., \& Valenti, J. 2005, ApJ, 622, 1102

Geiss, J. 1998, Space Sci. Rev., 85, 241

Gonzalez, G. 1997, MNRAS, 285, 403

Gonzalez, G. 2011, MNRAS, 416, L80

Gonzalez, G., Carlson, M. K., \& Tobin, R. W. 2010, MNRAS, 407, 314

González Hernández, J. I., Israelian, G., Santos, N. C., et al. 2010, ApJ, 720, 1592

González Hernández, J. I., Israelian, G., Santos, N. C., et al. 2011, in IAU Symp. 276, eds. A. Sozzetti, M. G. Lattanzi, \& A. P. Boss, 422

González Hernández, J. I., Delgado-Mena, E., Sousa, S. G., et al. 2013, A\&A, 552, A6

Grenon, M. 1987, J. Astrophys. Astron., 8, 123

Haywood, M. 2008a, A\&A, 482, 673

Haywood, M. 2008b, MNRAS, 388, 1175

Haywood, M. 2009, ApJ, 698, L1

Haywood, M., Di Matteo, P., Lehnert, M. D., Katz, D., \& Gómez, A. 2013, A\&A, 560, A109

Israelian, G., Delgado Mena, E., Santos, N. C., et al. 2009, Nature, 462, 189

Lemasle, B., François, P., Piersimoni, A., et al. 2008, A\&A, 490, 613

Lemasle, B., François, P., Genovali, K., et al. 2013, A\&A, 558, A31

Meléndez, J., Asplund, M., Gustafsson, B., \& Yong, D. 2009, ApJ, 704, L66

Minchev, I., Chiappini, C., \& Martig, M. 2013, A\&A, 558, A9

Mordasini, C., Alibert, Y., Benz, W., \& Naef, D. 2009, A\&A, 501, 1161

Nordström, B., Andersen, J., Olsen, E. H., et al. 1999, Ap\&SS, 265, 235

Nordström, B., Mayor, M., Andersen, J., et al. 2004, A\&A, 418, 989

Ramírez, I., Meléndez, J., \& Asplund, M. 2009, A\&A, 508, L17

Ramírez, I., Meléndez, J., \& Asplund, M. 2014, A\&A, 561, A7

Raymond, J. C. 1999, Space Sci. Rev., 87, 55

Rocha-Pinto, H. J., Flynn, C., Scalo, J., et al. 2004, A\&A, 423, 517

Santos, N. C., Israelian, G., \& Mayor, M. 2001, A\&A, 373, 1019

Santos, N. C., Israelian, G., \& Mayor, M. 2004, A\&A, 415, 1153

Schönrich, R., \& Binney, J. 2009, MNRAS, 399, 1145

Schuler, S. C., Flateau, D., Cunha, K., et al. 2011, ApJ, 732, 55

Sellwood, J. A., \& Binney, J. J. 2002, MNRAS, 336, 785

Smith, V. V., Cunha, K., \& Lazzaro, D. 2001, AJ, 121, 3207

Sousa, S. G., Santos, N. C., Israelian, G., Mayor, M., \& Udry, S. 2011, A\&A, 533, A141

Wang, Y., \& Zhao, G. 2013, ApJ, 769, 4

Wielen, R., Fuchs, B., \& Dettbarn, C. 1996, A\&A, 314, 438

Wood, B. E., \& Linsky, J. L. 2006, ApJ, 643, 444

Pages 5 to 6 are available in the electronic edition of the journal at http://www . aanda. org

The Neptunian hosts also host Jovian and super-Earth like planets. 


\section{Appendix A}

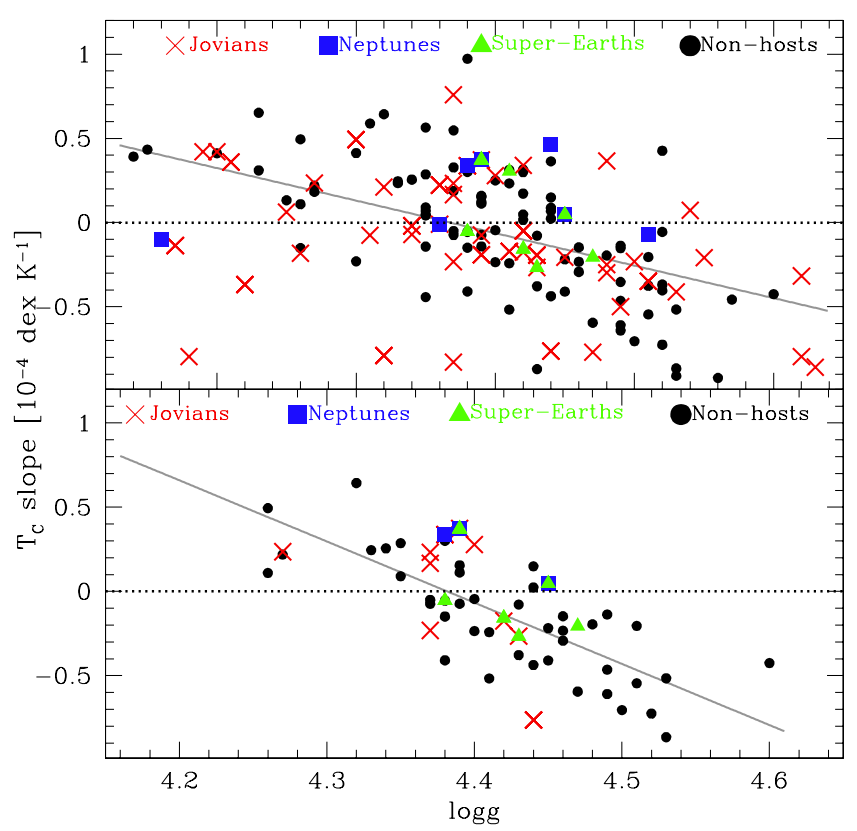

Fig. A.1. $T_{\mathrm{c}}$ slopes versus surface gravity of the stars from the full sample (top) and 58 solar analogs (bottom). Gray solid lines provide linear fits to the data points.
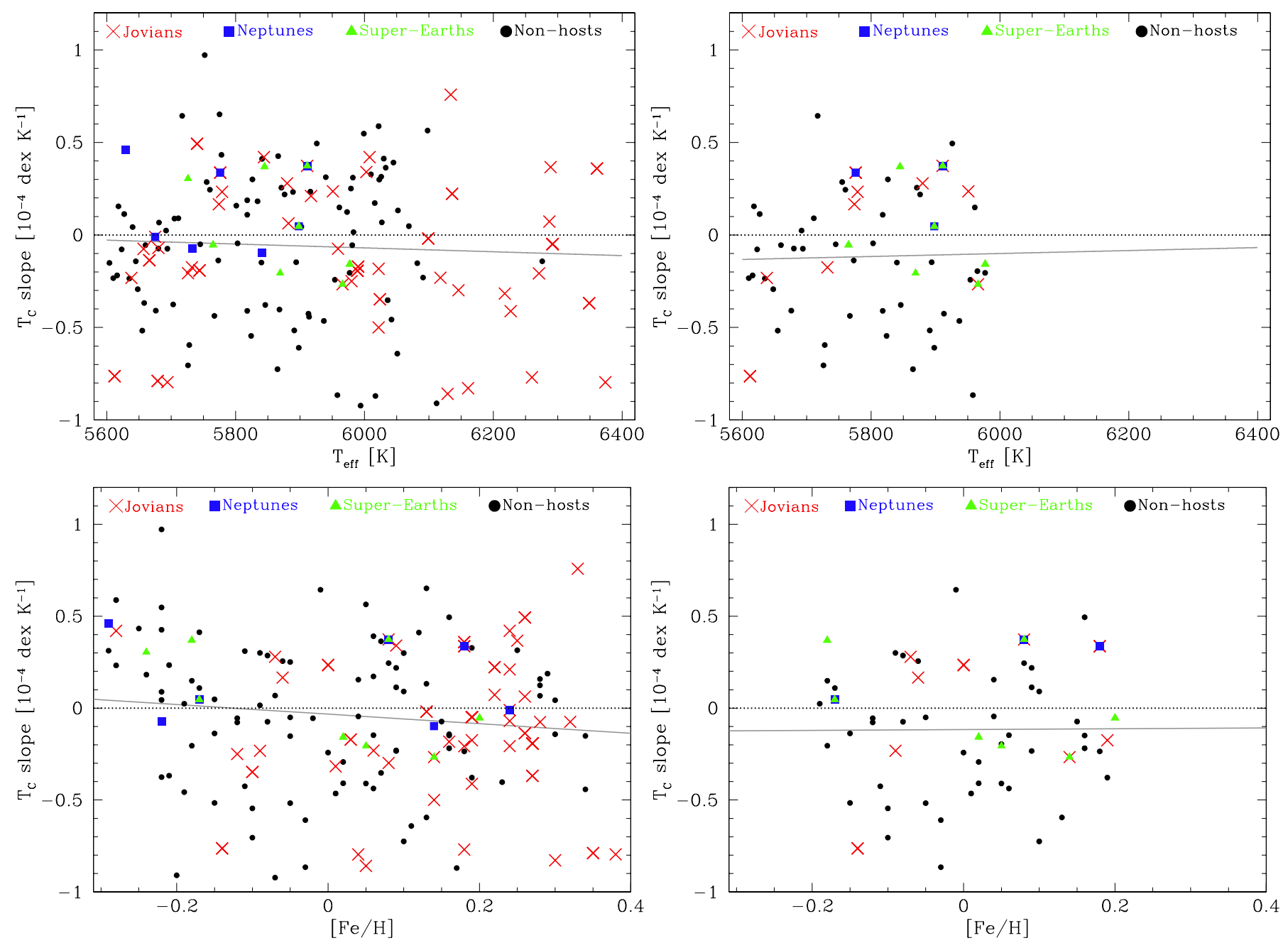

Fig. A.2. $T_{\mathrm{c}}$ slopes versus atmospheric parameters of the stars from the full sample (left) and solar analogs (right). 

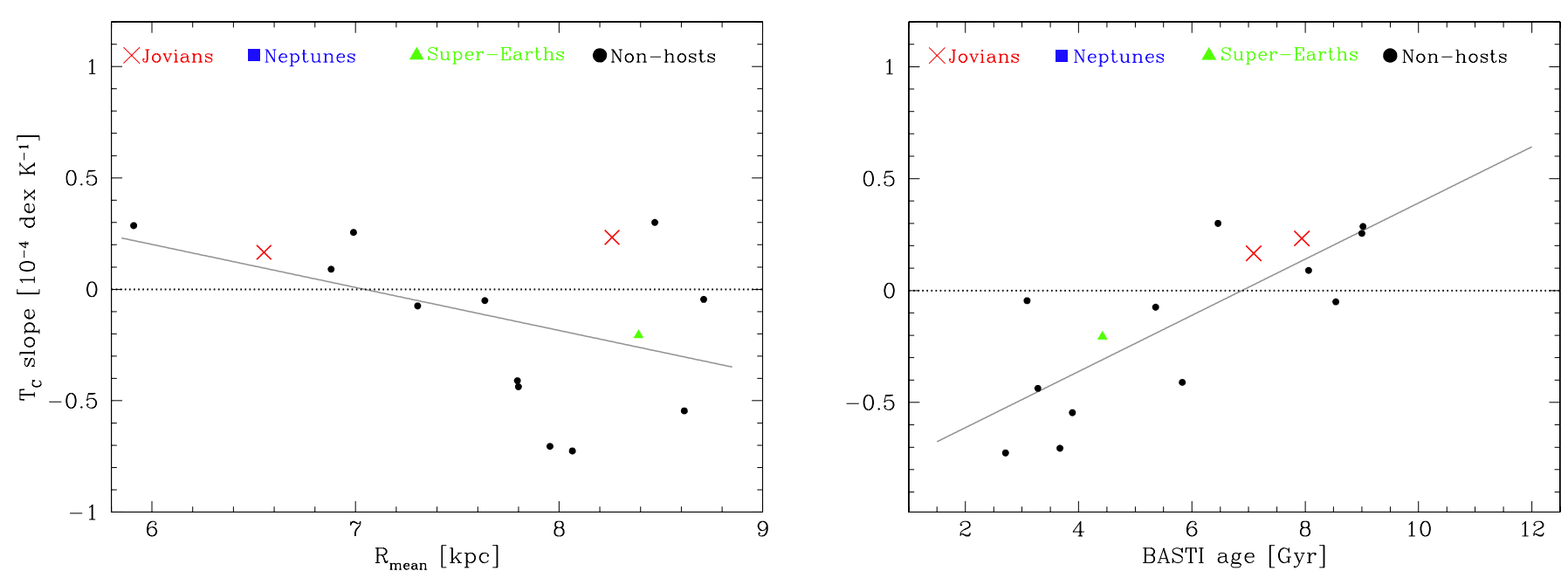

Fig. A.3. $T_{\mathrm{c}}$ slopes versus age and the mean of the apo- and pericentric distances for the solar twins in our sample. Gray solid lines provide linear fits to the data points.

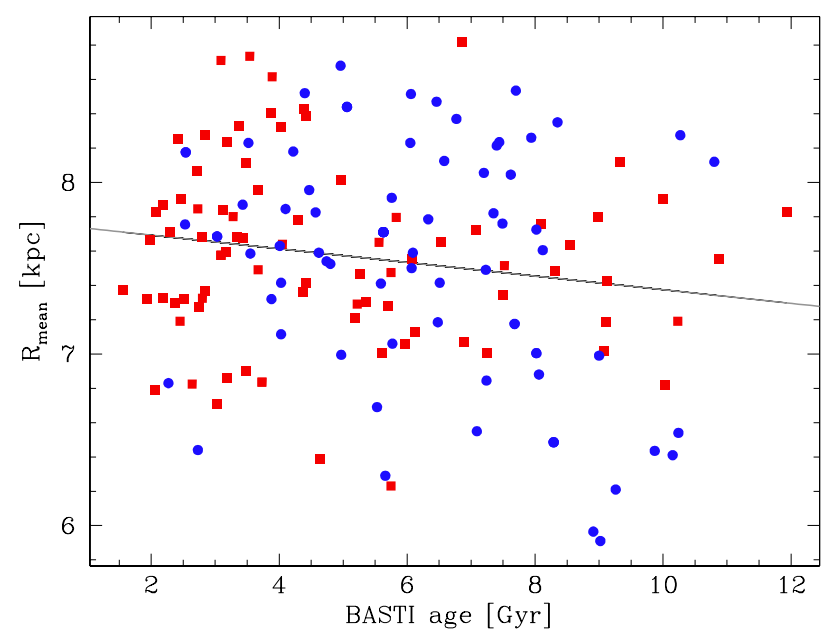

Fig. A.4. $R_{\text {mean }}$ versus age for the full sample. Red squares represent the stars with negative $T_{\mathrm{c}}$ slopes, and the stars with positive $T_{\mathrm{c}}$ slopes are marked by blue circles. Gray solid line provides linear fits to the full data points.

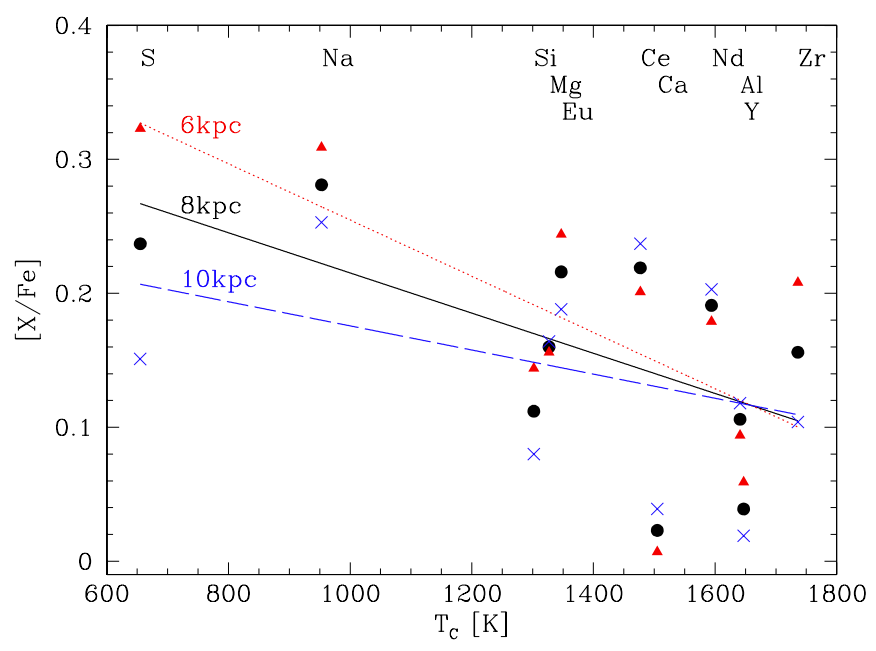

Fig. A.5. $[X / \mathrm{Fe}]$ versus $T_{\mathrm{c}}$ at three different galactocentric distances. The $[X / \mathrm{Fe}]$ values derived using the Galactic abundance gradients of Galactic Cepheids from Lemasle et al. $(2008,2013)$. The lines with different colors represent linear fits of the data. 